\title{
Langmuir
}

pubs.acs.org/Langmuir

(C) 2009 American Chemical Society

\section{All Solids, Including Teflon, Are Hydrophilic (To Some Extent), But Some Have Roughness Induced Hydrophobic Tendencies}

\author{
G. McHale* \\ School of Science and Technology, Nottingham Trent University, Clifton Lane, \\ Nottingham NG11 8NS, United Kingdom
}

Received February 17, 2009. Revised Manuscript Received May 17, 2009

\begin{abstract}
It has recently been argued on the basis of four experiments that Teflon can often be regarded as hydrophilic when considering the interaction between liquid water and the solid surface of Teflon [Gao and McCarthy, Langmuir 2008, 24, 9183-9188]. The authors also recommend that more recognition be given to "hydrophilic" and "hydrophobic" as qualitative adjectives and discuss the importance of advancing and receding contact angles. In this work, I use net surface free energy changes for events consisting of (i) a smooth solid wrapping a droplet of water and (ii) a grain attaching to a droplet, to show that all solids with a Young's law contact angle $\theta_{\mathrm{e}}<180^{\circ}$ can be considered absolutely hydrophilic. This terminology is true in the sense that attachment of the solid to the liquid is always preferred even though the relative strength decreases as $\theta_{\mathrm{e}}$ increases. However, I also demonstrate that, within a surface free energy model, solids with $\theta_{\mathrm{e}}>90^{\circ}$ can be regarded as possessing hydrophobic tendencies with increasing roughness. The effect of the bending rigidity of the substrate is discussed, and a condition for minimum droplet radius for wrapping to occur is given.
\end{abstract}

Gao and McCarthy have recently argued that under some circumstances Teflon can be regarded as hydrophilic. ${ }^{1}$ One of their experiments shows that a sufficiently thin film of Teflon contacted by a droplet of water will spontaneously wrap itself around the droplet. This type of spontaneous wrapping, termed capillary origami, has also been reported using polydimethylsiloxane (PDMS) membranes under the influence of evaporating droplets of water. ${ }^{2,3}$ In this Letter, I show from a surface free energy comparison why it is expected that all thin and smooth films of solids with a Young's law contact angle of less than $180^{\circ}$ should behave in this way, provided there is no energy cost to bending the substrate. I then relax this constraint and show that introducing a bending energy provides a simple criteria for wrapping involving the elasto-capillary number introduced by Bico and co-workers. ${ }^{3,4}$ I also argue that while all solids can be classified as absolutely hydrophilic on the basis of the wrapping criteria, they can also be divided into those with hydrophilic tendencies and those with hydrophobic tendencies on the basis of Wenzel's equation. 5,6 The use of the term "tendency" reflects the idea that the wetting behavior evolves toward either complete wetting or complete nonwetting, corresponding to Young's law contact angles of $0^{\circ}$ and $180^{\circ}$, respectively, as roughness increases providing intimate contact between the liquid and the solid is maintained.

Initially, consider a spherical droplet of liquid of radius $R$ and a solid shell of internal radius $R$ and thickness $\varepsilon$ (Figure 1a). In a vapor, the total surface free energy has three contributions: $F_{\text {initial }}=4 \pi R^{2} \gamma_{\mathrm{LV}}+4 \pi R^{2} \gamma_{\mathrm{SV}}+4 \pi(R+\varepsilon)^{2} \gamma_{\mathrm{SV}}$, where the $\gamma_{i j}$ are the interfacial tensions. Consider a final state in which the solid shell now encompasses the droplet so that the total surface free energy

*E-mail: glen.mchale@ntu.ac.uk. Telephone: + 44 (0)115 8483383.

(1) Gao, L.; McCarthy, T. J. Langmuir 2008, 24, 9183-9188.

(2) Py, C.; Reverdy, P.; Doppler, L.; Bico, J.; Roman, B.; Baroud, C. N. Phys. Rev. Lett. 2007, 98, 156103

(3) Py, C.; Reverdy, P.; Doppler, L.; Bico, J.; Roman, B.; Baroud, C. N. Eur. Phys. J. Spec. Top. 2009, 166, 67-71.

(4) Bico, J.; Roman, B.; Moulin, L.; Boudaoud, A. Nature 2004, 432, 690.

(5) Wenzel, R. N. Ind. Eng. Chem. 1936, 28, 988-994.

(6) Wenzel, R. N. J. Phys. Colloid Chem. 1949, 53, 1466-1467. has only two components: $F_{\text {final }}=4 \pi R^{2} \gamma_{\mathrm{SL}}+4 \pi(R+\varepsilon)^{2} \gamma_{\mathrm{SV}}$. The change in surface free energy between the final and initial states is then $\Delta F=F_{\text {final }}-F_{\text {initial }}=4 \pi R^{2}\left(\gamma_{\mathrm{SL}}-\gamma_{\mathrm{LV}}-\gamma_{\mathrm{SV}}\right)$, which upon using Young's law $\cos \theta_{\mathrm{e}}=\left(\gamma_{\mathrm{SV}}-\gamma_{\mathrm{SL}}\right) / \gamma_{\mathrm{LV}}$ gives

$$
\frac{\Delta F}{4 \pi R^{2} \gamma_{\mathrm{LV}}}=-\left(1+\cos \theta_{\mathrm{e}}\right)
$$

Since this is always negative for Young's law contact angles $\theta_{\mathrm{e}}<180^{\circ}$, all such solids reduce the energy of the droplet solid system by wrapping themselves around the droplet; only a solid with $\cos \theta_{\mathrm{e}}=\left(\gamma_{\mathrm{SV}}-\gamma_{\mathrm{SL}}\right) / \gamma_{\mathrm{LV}}<-1$ would be hydrophobic in the sense of not favoring any wrapping at all. In the case that a liquid completely wets a solid, the use of Young's law is not valid because the values of interfacial tensions effectively mean $\cos \theta_{\mathrm{e}}>1$ so that no equilibrium contact angle can be defined. However, in this case, $\Delta F<0$ is necessarily true.

In the preceding argument, the final shape with lowest energy has been taken as a sphere of water with a shell of the solid. In the case of just water, the sphere is the shape with the minimum surface area for a given volume. It is also the case that if we transfer a unit area section of a solid (assumed infinitesimally thin) wrapping the surface of a sphere of water into the bulk of the liquid (but without loss of surface area), we change the energy from $\gamma_{\mathrm{SL}}+\gamma_{\mathrm{SV}}$ to $\gamma_{\mathrm{LV}}+\gamma_{\mathrm{SL}}+\gamma_{\mathrm{SL}}$, which is a net change in surface free energy of $\Delta F=\gamma_{\mathrm{LV}}+\gamma_{\mathrm{SL}}-\gamma_{\mathrm{SV}}=\gamma_{\mathrm{LV}}\left(1-\cos \theta_{\mathrm{e}}\right)$. Since this change is positive, it is not energetically favorable to do so and so it is also not energetically favorable for the sphere with the solid to adopt a shape other than spherical with a shell of the solid (provided there is no energy stored in bending the solid and edge energies can be ignored).

Because of the assumption that the bending/crumpling of the solid does not cost energy, the use of a shell of solid could be replaced in this consideration by an initially flat film of surface area $4 \pi R^{2}$ or larger provided that the wrapped droplet only uses $4 \pi R^{2}$ of surface area and the remaining solid surface area remains in contact with the vapor. Thus, a thin film of any solid with no energy stored in bending (or crumpling) is hydrophilic in an 
a)
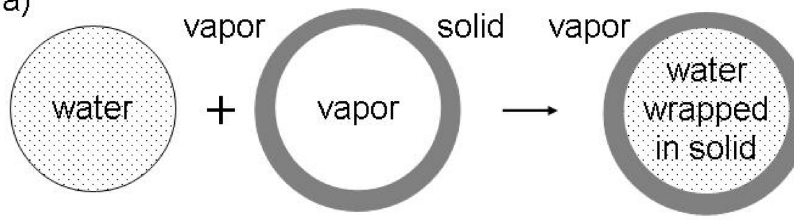

b)

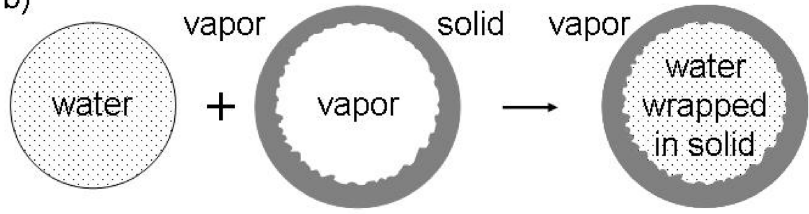

Figure 1. Initial and final states involved in a droplet wrapping event: (a) smooth solid shell and (b) solid shell with roughness, $r$. The droplet radius is $R$, and the solid shell has internal radius $R$ and external radius $(R+\varepsilon)$.

absolute sense although those with progressively higher values of $\theta_{\mathrm{e}}$ attach to water progressively less strongly as the magnitude of $\Delta F$ reduces. This concept of absolute hydrophilicity, with different relative strengths, includes solids, such as Teflon, with contact angles greater than $90^{\circ}$ as shown experimentally by McCarthy and Gao. ${ }^{1}$

A variation on this problem is to consider a solid possessing surface roughness $r$. Assume that while the solid can be wrapped around the droplet with no energy stored in the bending/ crumpling process, its surface roughness remains unchanged on contact with the liquid and that the liquid contacts that roughness at all points (Figure 1b). In this case, the solid-vapor interface on the inside of the shell has an area $4 \pi R^{2} r$ and this interface is replaced by a solid-liquid interface by the wrapping process. Equation 1 then becomes

$$
\frac{\Delta F}{4 \pi R^{2} \gamma_{\mathrm{LV}}}=-\left(1+r \cos \theta_{\mathrm{e}}\right)=-\left(1+\cos \theta_{\mathrm{W}}\right)
$$

where Wenzel's equation has been used; ${ }^{5,6}$ in this form, the magnitude of $\cos \theta_{\mathrm{W}}$ can be greater than unity. Equation 2 will be positive whenever $\theta_{\mathrm{e}}>90^{\circ}$ and $\left|r \cos \theta_{\mathrm{e}}\right|>1$, thus making the wrapping transition energetically unfavorable. Thus, the threshold defined by Wenzel's equation of $\theta_{\mathrm{e}}$ being lesser or greater than $90^{\circ}$ remains relevant for consideration of whether roughness will drive the system to the wrapping transition or away from it. In that sense, while a solid, such as Teflon, with $\theta_{\mathrm{e}}>90^{\circ}$ is hydrophilic in an absolute sense, its tendency with roughness is toward hydrophobic (defined here as complete nonwetting), and when $r \cos \theta_{\mathrm{e}}<-1$, is satisfied it becomes hydrophobic in an absolute sense.

It might also be argued on the basis of spontaneous penetration into a capillary that $\theta_{\mathrm{e}}=90^{\circ}$ is a natural threshold to separate the definition of solids between hydrophobic and hydrophilic. However, $\theta_{\mathrm{e}}=90^{\circ}$ is only the critical contact angle for capillary penetration when the channel is a parallel sided system. In the case of inclined or curved walls, the critical contact angle for capillary penetration can be significantly less than $90^{\circ} .^{7}$ Thus, using such a criterion as a definition of hydrophobicity or hydrophilicity would introduce a dependence on the orientation and/or geometry of the solid rather than simply relating it to the surface chemistry through the interfacial tensions. Similarly, the partial

(7) Shirtcliffe, N. J.; McHale, G.; Newton, M. I.; Pyatt, F. B.; Doerr, S. H. Appl. Phys. Lett. 2006, 89, 094101. wetting state of droplets usually observed on solids can be seen to be a consequence of both the surface chemistry and the rigidity of the solid. Moreover, the derivation of Young's law can also be seen to require the implicit assumption that the solid cannot bend under the action of surface tension; that is, the liquid always conforms to the solid shape, which itself is fixed by infinite rigidity.

To examine the consequence of rigidity of the solid (and so relax the assumption in the previous considerations of no energy being stored in bending), consider the energy for a solid spherical shell with a zero spontaneous curvature parameter,

$$
E_{\text {sphere }}=4 \pi\left(2 \kappa_{\mathrm{b}}+\kappa_{\mathrm{G}}\right)
$$

where $\kappa_{\mathrm{b}}$ is the bending rigidity and $\kappa_{\mathrm{G}}$ is the Gaussian bending rigidity; this type of problem involving curvature elasticity is well-known within the mechanics of the cell. ${ }^{8}$ An alternative and simpler situation, not considered in detail here, would be to consider the wrapping of a cylinder of a liquid so that the Gaussian bending rigidity would not be relevant. Using eq 2 and assuming any edge energy associated with a flat plate is zero, the condition for wrapping of the solid around the liquid droplet can then be written as

$$
-R^{2} \gamma_{\mathrm{LV}}\left(1+\cos \theta_{\mathrm{W}}\right)+\left(2 \kappa_{\mathrm{b}}+\kappa_{\mathrm{G}}\right)<0
$$

Defining an elasto-capillary bending length, $L_{\mathrm{EC}}=\left(\kappa_{\mathrm{b}} / \gamma_{\mathrm{LV}}\right)^{1 / 2}$ (which was first defined in ref 4), and a Gaussian-capillary bending length, $L_{\mathrm{GC}}=\left(\kappa_{\mathrm{b}} / \gamma_{\mathrm{LV}}\right)^{1 / 2}$, gives the condition for wrapping of a droplet of radius $R$ to occur as

$$
\cos \theta_{\mathrm{W}}>-1+\frac{\left(2 L_{\mathrm{EC}}{ }^{2}+L_{\mathrm{GC}}{ }^{2}\right)}{R^{2}}
$$

When the rigidity of a solid is infinitely large, eq 5 implies wrapping cannot occur. Under these circumstances, a droplet of liquid placed on a solid surface cannot change the solid shape. This is the condition implicitly assumed in the derivation of Young's law, and this means that a liquid is forced to change its shape under the action of the interfacial forces as it tries to conform to the substrate shape defined by the solid. When the bending rigidity terms (i.e., curvature energy) vanish, eq 5 reduces to the condition given by eq 2 that assumed no energy was stored in the rigidity effect of a change of shape in the wrapping of the solid; the solid can conform to the shape defined by the liquid. This latter situation may seem an extreme one to use to define a solid as being in an absolute sense hydrophilic, but it essentially removes rigidity from the definition of whether a solid is hydrophilic or hydrophobic and leaves only a consideration of the interfacial tensions. In my view, it can certainly be argued that to include the degree of rigidity of a solid in the definition of hydrophilic and hydrophobic would seem strange.

When a droplet becomes sufficiently small, eq 5 cannot be satisfied and the bending rigidity prevents the wrapping from occurring. The critical droplet radius, $R_{\mathrm{c}}$, from eq 5 is given by

$$
R_{\mathrm{c}}=\sqrt{\frac{2 L_{\mathrm{EC}}^{2}+L_{\mathrm{GC}}{ }^{2}}{1+\cos \theta_{W}}}
$$

(8) Boal, D. H. Mechanics of the Cell, 1st ed.; Cambridge University Press: New York, 2002 
In the case of a complete wetting liquid $\left(\theta_{\mathrm{e}}=0^{\circ}\right)$ on a smooth thin plate of thickness $h$ with a vanishing Gaussian rigidity and the bending rigidity given by $\kappa_{\mathrm{b}}=E h^{3} / 12\left(1-v^{2}\right)$, where $E$ is Young's modulus and $v$ is Poisson's ratio, the critical droplet radius is $R_{\mathrm{c}}=L_{\mathrm{EC}}$. This result can be compared for the critical radius, $R_{\mathrm{c}}=L_{\mathrm{EC}} / \sqrt{ } 2$, for the wrapping of a cylindrical droplet given Py et al., ${ }^{2,3}$ As observed in the experiments of Gao and McCarthy, ${ }^{1}$ wrapping of a droplet by a solid film results in a nonspherical shape due to energies associated with the bending, crumpling, folding, and stretching of the solid.

The practicalities in making films that can spontaneously selfwrap around a droplet can be quite severe, particularly if the solid is rough and that roughness has to be maintained after the wrapping has occurred; a practical example of a solid with hard roughness might be a flexible solid sheet with embedded particles. However, the wrapping of a film of a solid around a droplet of water has many similarities to the formation of a liquid marble, , $^{9}$ which was another of the experimental systems referred to by Gao and McCarthy as showing why "hydrophobic particles" are relatively hydrophilic in the sense of possessing a positive attraction to water. ${ }^{1}$ Taking a spherical grain of radius $R_{\mathrm{g}}$ and roughness $r$, the change in surface free energy when it attaches to

(9) Aussillous, P.; Quéré, D. Nature (London) 2001, 411, 924-927.

(10) McHale, G.; Herbertson, D. L.; Elliott, S. J.; Shirtcliffe, N. J.; Newton, M. I. Langmuir 2007, 23, 918-924. a droplet of radius $R$ is

$$
\begin{aligned}
\frac{\Delta F}{4 \pi R_{\mathrm{g}}{ }^{2} \gamma_{\mathrm{LV}}} & =-\left(1+\cos \theta_{\mathrm{e}}\right)\left(1+r \cos \theta_{\mathrm{e}}\right) \\
& =-\left(1+\cos \theta_{\mathrm{e}}\right)\left(1+\cos \theta_{\mathrm{W}}\right)
\end{aligned}
$$

Thus, a smooth $(r=1)$ hydrophobic spherical grain will attach to a droplet whenever the Young's law contact angle satisfies $\theta_{\mathrm{e}}<180^{\circ}$, but a grain would not attach if $\theta_{\mathrm{e}}>90^{\circ}$ and its roughness was sufficiently large such that $\left|r \cos \theta_{\mathrm{e}}\right|>1$. A collection of grains assembling to form a liquid marble resembles a solid with zero rigidity (a liquidlike solid) wrapping a droplet of a liquid. From this perspective, all solids are hydrophilic in an absolute sense (ignoring energies associated with the rigidity of the solid), but those with $\theta_{\mathrm{e}}>90^{\circ}$ have hydrophobic tendencies.

None of the above considerations address the very valid point that real solids, such as Teflon, generally exhibit advancing and receding contact angles rather than the theoretical Young's law contact angle or that contact angle hysteresis determines the ability of a surface to shed an attached droplet. A high observed contact angle, which is often referred to in the literature as superhydrophobic, and the droplet shedding ability of a surface are not necessarily the same, a point made very elegantly by ref 1 .

Acknowledgment. The author acknowledges useful discussions with Professsor T. J. McCarthy. 